KCL-MTH-98-59

hep-th/9811178

November 1998

\title{
Boundary structure constants for the A-series Virasoro minimal models
}

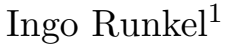 \\ Mathematics Department, \\ King's College London, Strand, London WC2R 2LS, U.K.
}

\begin{abstract}
We consider A-series modular invariant Virasoro minimal models on the upper half plane. From Lewellen's sewing constraints a necessary form of the bulk and boundary structure constants is derived. Necessary means that any solution can be brought to the given form by a rescaling of the fields. All constants are expressed essentially in terms of fusing (F-)matrix elements and the normalisations are chosen such that they are real and no square roots appear. It is not shown in this paper that the given structure constants solve the sewing constraints, however random numerical tests show no contradiction and agreement of the bulk structure constants with Dotsenko and Fateev. In order to facilitate numerical calculations a recursion relation for the F-matrices is given.
\end{abstract}

\footnotetext{
${ }^{1}$ e-mail: ingo@mth.kcl.ac.uk
} 


\section{Introduction}

Conformal field theory (CFT) arises in the description of the long range behaviour of statistical systems at a critical point. In two dimensions the symmetry algebra of the CFT becomes infinite dimensional and places severe constraints on the theory. From the point of view of statistical mechanics it is natural to consider systems with boundaries instead of infinitely extended systems. Introducing the boundary reduces the symmetry of the system, but for certain boundary conditions an infinite subset of the original symmetries remains unbroken. This leads to a boundary conformal field theory (BCFT).

Virasoro minimal models (see e.g. [1,2]) are an important subclass of two dimensional CFTs. [3] gives an A-D-E type classification of the field content of these models in the bulk. To know all correlation functions it is sufficient to know the conformal blocks (which can be found as solutions to linear differential equations) and the structure constants which are determined by duality properties of the correlators. The structure constants are of interest themselves, e.g. as an essential ingredient in TCSA ( [4, 5]). For a CFT restricted to the upper half plane one has to know four sets of constants: the structure constants appearing in the operator product expansion (OPE) of bulk fields, the structure constants of boundary fields, how bulk fields couple to boundary fields and the one point functions of the identity on different boundaries.

The classification of the field content and possible boundary conditions of BCFTs was initiated by Cardy [6, ,7] and further addressed e.g. in [8 10]. Once the field content of a BCFT is known, there is a set of consistency conditions, the sewing constraints (see [11]), which the structure constants of the theory have to obey. The main ingredient in the constraint equations are the matrices that transform between different bases of conformal blocks, which are called F-matrices (see e.g [12]). One can now try to use the sewing constraints to determine the structure constants, and in the case of A-type Virasoro minimal models it turns out that they can be expressed essentially in terms of F-matrix elements. Explicit expressions for the bulk structure constants can be found in [13] and the bulk-boundary couplings have been derived in [7]. In this paper only A-invariant Virasoro minimal models on the upper half plane are considered and for these the remaining structure constants are derived. Care has been taken to give the expressions in a form that facilitates numerical computation, e.g. no square roots appear and all results are real. It is not proven in this paper that the structure constants given solve the complete set of sewing constraints 1 , but random numerical tests showed no contradiction.

Section 2 sets the conventions used for conformal blocks and section 3 gives a recursion formula to compute the F-matrices. In section 10 some results about the classification of boundary conditions and field content are stated and the notation for boundary fields is established. Section 5 gives Lewellen's sewing constraints in the diagonal case. These are used in sections 6 and 0 to compute necessary forms of all structure constants. The implications for the identity-1-point functions of consistency under modular transformation and continuity on the disc are studied in section 8 .

\footnotetext{
${ }^{1}$ In this paper only constraints arising for a CFT on the upper half plane are considered. For sewing constraints on non-orientable surfaces see [14.
} 


\section{Transformation of Conformal Blocks}

First we introduce some notation: Let $\mathrm{M}\left(p^{\prime}, p\right)$ denote an A-invariant Virasoro minimal model. It contains only spinless primary fields $\phi_{i}$ with conformal weights $h_{i}=\bar{h}_{i}$. The weights $h_{i}=h_{(r, s)}$ take values in the Kac-table:

$$
h_{(r, s)}=\frac{1}{4 t}\left(d^{2}-(t-1)^{2}\right) \quad \text { with } \quad d=r \cdot t-s \quad ; \quad t=p / p^{\prime}
$$

The indices $r, s$ run over $1 \leq r<p^{\prime}$ and $1 \leq s<p$. Each conformal weight appears twice. For example $\mathrm{M}(5,2)$ has $t=2 / 5$ and contains two independent fields $\phi_{(1,1)}=\phi_{(4,1)}$ and $\phi_{(2,1)}=\phi_{(3,1)}$ with conformal weights $h_{(1,1)}=h_{(4,1)}=0$ and $h_{(2,1)}=h_{(3,1)}=-1 / 5$.

In a RCFT without boundaries, correlators can be expressed as a finite sum of products of holomorphic and antiholomorphic functions, e.g. for the 4-pt-functions:

$$
\left\langle i\left|\phi_{j}(z, \bar{z}) \phi_{k}(w, \bar{w})\right| \ell\right\rangle=\sum_{a, b}^{N<\infty} c_{a b} f_{a}(z, w) \bar{f}_{b}(\bar{z}, \bar{w})
$$

The functions $f_{k}(z, w)$ have to obey a certain set of linear differential equations resulting from zero norm states in the Hilbert space. Let $V_{i \ell}^{j k}$ denote the vector space of all such functions.

We will now choose two different bases in $V_{i \ell}^{j k}$. The first one is associated with the asymptotic behaviour of $f(z, w)$ as $w \rightarrow 0$. Following [12] we introduce a pictorial notation:

$$
\frac{\left.i{ }^{j} p\right|^{k} \ell}{(z)(w)}:=f(z, w) \quad \text { if for } w \rightarrow 0: \quad f(1, w)=w^{h_{p}-h_{k}-h_{\ell}}(1+\cdots)
$$

The second basis is given by the behaviour as $w \rightarrow z$ :

$$
\begin{array}{l|l}
q & j \\
i \quad \begin{array}{c}
q-w) \\
\ell
\end{array} & \quad \text { if for } w \rightarrow 1: \quad g(1, w)=(1-w)^{h_{q}-h_{j}-h_{k}}(1+\cdots)
\end{array}
$$

These are just the conformal blocks as given e.g. in [12] normalisation. The number of independent 3-pt couplings of representation $i, j, k$ is given by the Verlinde fusion numbers $\mathrm{N}_{i j}{ }^{k}$ which are either 0 or 1 for Virasoro minimal models. The number of blocks of type (阿), (荬) are $\mathrm{N}_{i j}{ }^{p} \cdot \mathrm{N}_{k l}{ }^{p}$ and $\mathrm{N}_{j k}{ }^{q} \cdot \mathrm{N}_{i l}{ }^{q}$ respectively and hence are also either 0 or 1 .

The fusing matrix $\mathrm{F}$ is defined as a transformation between the two different bases of conformal blocks:

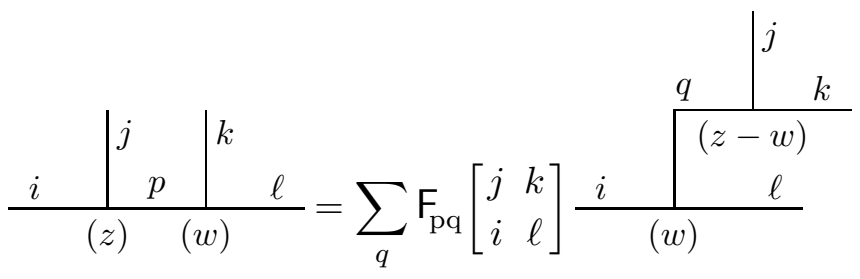

\footnotetext{
${ }^{2}$ In [12] conformal blocks are defined in a more general setting using vertex operators.
} 
From the explicit form of the 3-point function one can check that the fusion matrix is equal to 1 if any of the $i, j, k, \ell$ is the identity and the resulting 3-point function is nonzero.

\section{A Recursion Formula for F-Matrices}

The F-matrices depend on the normalisation of the conformal blocks. In the present normalisation the complicated expressions in terms of gamma functions are hidden inside the F-matrix entries and the expressions for the structure constants are in turn very easy. In another normalisation the F-matrices are just the quantum $6 \mathrm{j}$-symbols (see e.g. [15, 16]) and it is in principle possible to relate the two normalisations by using expressions given in 13. In the present paper a different approach has been taken, that is the F-matrices are computed recursively. The recursion formula given below has the advantages that it is easy to derive, doesn't cause any problems for rational values of the central charge, is manifestly real and doesn't introduce sign problems through square roots (for another recursion relation derived in a similar way see [17]).

To obtain the starting point of the recursion one can solve explicitly the differential equation for the 4-pt-function $\left\langle i\left|\phi_{j}(1) \phi_{(2,1)}(x)\right| \ell\right\rangle$ (see e.g. [2, 13]). This results in a sum involving hypergeometric functions from which the F-matrix can be read off. The Fmatrix for the $(1,2)$ field can be obtained from the isomorphism between $\mathrm{M}\left(p^{\prime}, p\right)$ and $\mathrm{M}\left(p, p^{\prime}\right)$, which amounts to the replacement $d \rightarrow-t^{-1} d$.

In the following formula $\Delta$ stands for either $(2,1)$ or $(1,2)$. If $j$ stands for $(r, s)$ then $j \pm \Delta$ is $(r \pm 1, s)$ and $(r, s \pm 1)$ respectively. Altogether one gets:

$$
\mathrm{F}\left[\begin{array}{cc}
j & \Delta \\
i & \ell
\end{array}\right]=\left(\begin{array}{ll}
\mathrm{F}_{\ell-\Delta, j-\Delta} & \mathrm{F}_{\ell-\Delta, j+\Delta} \\
\mathrm{F}_{\ell+\Delta, j-\Delta} & \mathrm{F}_{\ell+\Delta, j+\Delta}
\end{array}\right)=\cdots
$$

for $\Delta=(2,1)$ :

$$
=\left(\begin{array}{cc}
\frac{\Gamma\left(d_{j}\right) \Gamma\left(1-d_{\ell}\right)}{\Gamma\left(\frac{1}{2}\left(1-d_{i}+d_{j}-d_{\ell}\right)\right) \Gamma\left(\frac{1}{2}\left(1+d_{i}+d_{j}-d_{\ell}\right)\right)} & \frac{\Gamma\left(-d_{j}\right) \Gamma\left(1-d_{\ell}\right)}{\Gamma\left(\frac{1}{2}\left(1-d_{i}-d_{j}-d_{\ell}\right)\right) \Gamma\left(\frac{1}{2}\left(1+d_{i}-d_{j}-d_{\ell}\right)\right)} \\
\frac{\Gamma\left(d_{j}\right) \Gamma\left(1+d_{\ell}\right)}{\Gamma\left(\frac{1}{2}\left(1-d_{i}+d_{j}+d_{\ell}\right)\right) \Gamma\left(\frac{1}{2}\left(1+d_{i}+d_{j}+d_{\ell}\right)\right)} & \frac{\Gamma\left(-d_{j}\right) \Gamma\left(1+d_{\ell}\right)}{\Gamma\left(\frac{1}{2}\left(1-d_{i}-d_{j}+d_{\ell}\right)\right) \Gamma\left(\frac{1}{2}\left(1+d_{i}-d_{j}+d_{\ell}\right)\right)}
\end{array}\right)
$$

for $\Delta=(1,2)$ :

$$
=\left(\begin{array}{cc}
\frac{\Gamma\left(-\frac{1}{t} d_{j}\right) \Gamma\left(\frac{1}{t}\left(t+d_{\ell}\right)\right)}{\Gamma\left(\frac{1}{2 t}\left(t+d_{i}-d_{j}+d_{\ell}\right)\right) \Gamma\left(\frac{1}{2 t}\left(t-d_{i}-d_{j}+d_{\ell}\right)\right)} & \frac{\Gamma\left(\frac{1}{t} d_{j}\right) \Gamma\left(\frac{1}{t}\left(t+d_{\ell}\right)\right)}{\Gamma\left(\frac{1}{2 t}\left(t+d_{i}+d_{j}+d_{\ell}\right)\right) \Gamma\left(\frac{1}{2 t}\left(t-d_{i}+d_{j}+d_{\ell}\right)\right)} \\
\frac{\Gamma\left(-\frac{1}{t} d_{j}\right) \Gamma\left(\frac{1}{t}\left(t-d_{\ell}\right)\right)}{\Gamma\left(\frac{1}{2 t}\left(t+d_{i}-d_{j}-d_{\ell}\right)\right) \Gamma\left(\frac{1}{2 t}\left(t-d_{i}-d_{j}-d_{\ell}\right)\right)} & \frac{\Gamma\left(\frac{1}{t} d_{j}\right) \Gamma\left(\frac{1}{t}\left(t-d_{\ell}\right)\right)}{\Gamma\left(\frac{1}{2 t}\left(t+d_{i}+d_{j}-d_{\ell}\right)\right) \Gamma\left(\frac{1}{2 t}\left(t-d_{i}+d_{j}-d_{\ell}\right)\right)}
\end{array}\right)
$$

where $d_{i}, d_{j}$ and $d_{\ell}$ are defined as in (11). Depending on the fusion rules between $i, j, \Delta, \ell$ none or only one of the above matrix elements may be allowed.

Using the techniques introduced in 12] on 5-point-functions, or by deriving it directly from the pentagon identity, one can obtain a recursive formula for the F-matrices:

$$
\mathrm{F}_{\mathrm{pq}}\left[\begin{array}{cc}
j & k+\Delta \\
i & \ell
\end{array}\right]=\sum_{r, s} \mathrm{~F}_{\mathrm{k}+\Delta, \mathrm{r}}\left[\begin{array}{cc}
\ell & \Delta \\
p & k
\end{array}\right] \cdot \mathrm{F}_{\mathrm{ps}}\left[\begin{array}{cc}
j & k \\
i & r
\end{array}\right] \cdot \mathrm{F}_{\mathrm{rq}}\left[\begin{array}{cc}
s & \Delta \\
i & \ell
\end{array}\right] \cdot \mathrm{F}_{\mathrm{s}, \mathrm{k}+\Delta}\left[\begin{array}{cc}
\Delta & k \\
q & j
\end{array}\right]
$$


The recursion runs on the index $k$ of $\mathrm{F}_{p q}[i \ell ; j k]$. In each step (6) gives the F-matrix elements for a fixed $k$ and arbitrary (allowed) $i, j, \ell, p, q$. Knowing all these on can proceed to $k \rightarrow k+\Delta$.

The index range of the sum in (6) is determined through the requirement that the eight diagrams associated to the four $\mathrm{F}$-matrices have to exist. The independent conditions on $r, s$ are: $\mathrm{N}_{p k}^{r} \cdot \mathrm{N}_{l \Delta}^{r} \neq 0, \mathrm{~N}_{j k}^{s} \cdot \mathrm{N}_{q \Delta}{ }^{s} \neq 0$ and $\mathrm{N}_{r s}^{i} \neq 0$.

In particular the maximal range of the summation indices is $r=\ell \pm \Delta$ and $s=q \pm \Delta$. Depending on $i, j, k, p$ it may however be smaller than that.

\section{Boundary Conditions and Field Content}

A boundary conformal field theory (BCFT) on the upper half plane (UHP) is specified by the field content in the bulk $\phi_{i}$, the possible boundary conditions which preserve conformal symmetry $a$ and the boundary fields $\psi_{i}^{(a b)}$ that interpolate boundary conditions $a$ and $b$, as well as the structure constants in OPEs of all these fields. Note that the boundary conditions $a, b$ to the left and right of $x$ in $\psi_{i}^{(a b)}(x)$ may or may not be different and one can interpret the field as a boundary changing field or a field that lives on a certain boundary, respectively.

On the UHP there is always the possibility of a boundary field inserted at infinity. If the boundary conditions towards left and right infinity are different this has to be the case, and if they equal there may or may not be a field at infinity. The situation is clearer when we map the UHP to the unit disc. Any insertion at infinity in the UHP will appear as an additional boundary field on the unit circle. From hereon we will only consider situations in the UHP which, when mapped to the disc via some Möbius transformation $z \mapsto \varphi(z)$, have no boundary field at $\varphi(\infty)$. In particular on the UHP this implies that we have same boundary condition towards $\pm \infty$ and no boundary field inserted at $\infty$. This is not a restriction, because the situation just described can be related to the general case on the UHP, i.e. the case with fields inserted at infinity and possibly different boundary conditions towards left and right infinity, via transforming it to the unit disc, rotating the disc and mapping it back to the UHP.

There are three different OPEs to consider: Two bulk fields coming together, expanding a bulk field in terms of boundary fields, and two boundary fields coming together. When the bulk fields are spinless these are, in turn l:

$$
\begin{aligned}
\phi_{i}(z) \phi_{j}(w) & \sim \sum_{k} C_{i j}{ }^{k} \cdot \phi_{k}(w) \cdot|z-w|^{2\left(h_{k}-h_{i}-h_{j}\right)} \\
\phi_{i}(x+i y) & \sim \sum_{k}{ }^{(a)} B_{i}{ }^{k} \cdot \psi_{k}^{(a a)}(x) \cdot(2 y)^{h_{k}-2 h_{i}} \\
\psi_{i}^{(a b)}(x) \psi_{j}^{(b c)}(y) & \sim \sum_{k} C_{i j}^{(a b c) k} \cdot \psi_{k}^{(a c)}(y) \cdot(x-y)^{h_{k}-h_{i}-h_{j}} \quad ; x>y
\end{aligned}
$$

\footnotetext{
${ }^{3}$ Note that there is a slight difference to the notation used by Lewellen in [11]. There the boundary $\mathrm{OPE}$ is $\psi_{i}^{(a b)}(x) \psi_{j}^{(b c)}(y) \sim \sum_{k} C_{i j}^{(a b c) k} \psi_{k}^{(a c)}(x) \cdot(y-x)^{h_{k}-h_{i}-h_{j}}$. The difference is in the ordering of the boundary fields in the n-point-functions. For the 3-pt-function the precise correspondence is, with $x_{1}>x_{2}>x_{3}:\left\langle\psi_{i}^{(a b)}\left(x_{1}\right) \psi_{j}^{(b c)}\left(x_{2}\right) \psi_{k}^{(c a)}\left(x_{3}\right)\right\rangle_{\text {here }}=\left\langle\psi_{k}^{(a c)}\left(x_{3}\right) \psi_{j}^{(c b)}\left(x_{2}\right) \psi_{i}^{(b a)}\left(x_{1}\right)\right\rangle_{\text {Lew. }}$, so that the relation between structure constants is $C_{i j}^{(a b c) k}$ (here) $=C_{j i}^{(c b a) k}$ (Lew.). But that doesn't actually matter, because it will turn out that the explicit form given later fulfils $C_{i j}^{(a b c) k}=C_{j i}^{(c b a) k}$.
} 
The boundary conditions of diagonal modular invariants are given in [7]. A more general classification including non-diagonal theories can be found e.g. in 8 10]. Here we collect some of their results we will need in the following:

For a diagonal theory the possible boundary conditions are labelled by the bulk fields. The partition function on a cylinder of length $R$ and circumfence $L$ with boundary conditions $a$ and $b$ is given by:

$$
Z_{(a b)}=\sum_{i} n_{i a}^{b} \cdot \chi_{i}(q) \quad ; q=e^{-\pi \frac{L}{R}}
$$

In a diagonal theory the $n_{i a}{ }^{b}$ are just the Verlinde fusion numbers $n_{i a}{ }^{b}=\mathrm{N}_{a b}{ }^{i}$. In particular only the identity field lives on the 1-boundary: $n_{i 1}{ }^{1}=\delta_{i 1}$, and only the field $\psi_{a}^{(1 a)}$ can interpolate the 1 - and $a$-boundary condition: $n_{i 1}{ }^{a}=\delta_{i a}$. Furthermore all bulk fields couple to the identity on the 1-boundary: ${ }^{(1)} B_{i}{ }^{1} \neq 0 \forall i$.

\section{Sewing Constraints for Diagonal Models}

In 111 Lewellen gives a complete set of consistency conditions for the structure constants of a Virasoro minimal model on the UHP . In the diagonal case one can, up to rescaling, determine the necessary form of a solution to these conditions by only considering the subset of these conditions, as given below (1 denotes the identity field):

For four boundary fields $i, j, k, \ell$ :

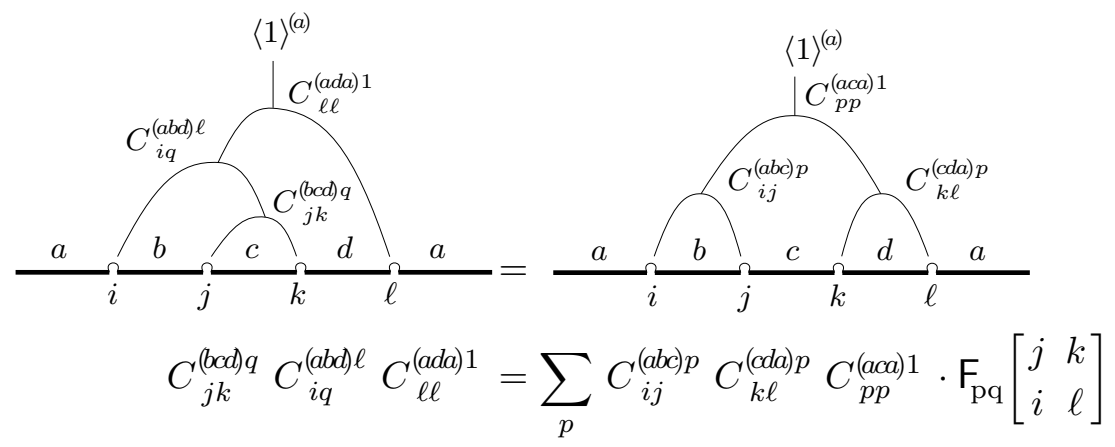

For one bulk field $i$ and two boundary fields $p, q$ :

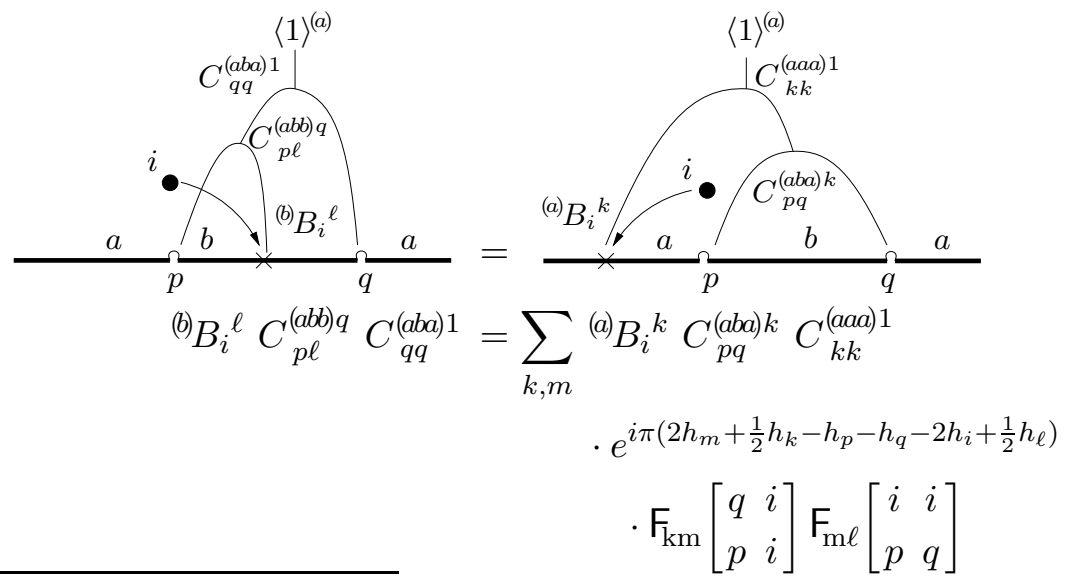

${ }^{4}$ The results in [8] differ from [11]. The equations given here were derived using methods from [12] to transform between the different bases of conformal blocks in the case where only diagonal fields are present and agree with [11]. We hope to clarify the relation to [8] in future work. 
For two bulk fields $i, j$ :

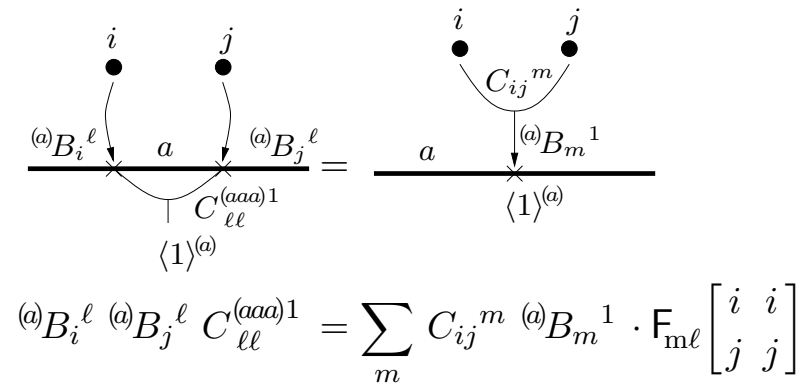

The remaining two sewing constraints, for completeness, are:

For two bulk fields and one boundary field:

$$
\begin{gathered}
{ }^{(a)} B_{k}{ }^{q(a)} B_{\ell}{ }^{t} C_{q t}^{(a a) i}=e^{i \frac{\pi}{2}\left(h_{t}-h_{i}-h_{q}-2 h_{\ell}\right)} \sum_{p, r} C_{k \ell^{p}}{ }^{(a)} B_{p}{ }^{i} e^{i \pi h_{r}} \\
\cdot \mathrm{F}_{\mathrm{pr}}\left[\begin{array}{ll}
i & \ell \\
p & k
\end{array}\right] \mathrm{F}_{\mathrm{pq}}\left[\begin{array}{ll}
\ell & r \\
k & k
\end{array}\right] \mathrm{F}_{\mathrm{rt}}\left[\begin{array}{ll}
\ell & \ell \\
q & i
\end{array}\right]
\end{gathered}
$$

For four bulk fields:

$$
C_{i j}{ }^{s} C_{k \ell}{ }^{s} C_{s s}{ }^{1} \mathrm{~F}_{\mathrm{st}}\left[\begin{array}{ll}
j & k \\
i & \ell
\end{array}\right]=C_{j k}{ }^{t} C_{\ell i}{ }^{t} C_{t t}{ }^{1} \mathrm{~F}_{\mathrm{ts}}\left[\begin{array}{ll}
\ell & k \\
i & j
\end{array}\right]
$$

These equations can be verified e.g. with the techniques for conformal blocks described in [12]. With the identities on F-Matrices given there it is also possible to see that, for real F, the RHS of (12) and (14) are equal to their complex conjugates and thus real.

Note that in the pictures associated to equations (11)-(13) the 1-pt-function of the identity field $\langle 1\rangle^{(a)}$ appears on the LHS and RHS. In the given form of the sewing constraints these have been cancelled from the equations itself, so that the structure constants computed from (11)-(13) do not depend on the 1-pt-functions of the 1-field.

We still have the freedom to rescale all the fields. This freedom must be reflected in (11)-(15) in that a rescaling of the fields maps a solution to a solution. When rescaling particular fields inside the n-pt-functions the structure constants have to change accordingly so that the expansions (7)-(9) remain valid. One can now verify that all possible rescalings map solutions of $(11)-(15)$ to solutions.

\section{Bulk-Boundary Couplings}

First we use the sewing constraints to fix the necessary form of the bulk-boundary couplings. The bulk fields can be rescaled $\phi_{i} \rightarrow \lambda \cdot \phi_{i}$ s.t. ${ }^{(1)} B_{i}{ }^{1}=S_{i}{ }^{1} / S_{1}{ }^{1}$ (recall that from [0] we know that ${ }^{(1)} B_{i}{ }^{1} \neq 0 \forall i$ ). Now consider (13) in the form:

$$
\sum_{\ell}{ }^{(a)} B_{i}^{\ell(a)} B_{j}^{\ell} C_{\ell \ell}^{(a a a) 1} \mathrm{~F}_{\ell \mathrm{n}}\left[\begin{array}{ll}
j & i \\
j & i
\end{array}\right]=C_{i j}{ }^{n(a)} B_{n}{ }^{1}
$$


Since by assumption only $\psi_{1}^{(11)}$ can exist on the 1-boundary, for $a=1$ the sum reduces to one term $\ell=1$. Using (48) we obtain:

$$
C_{i j}{ }^{k}=\frac{{ }^{(1)} B_{i}{ }^{1}{ }^{(1)} B_{j}{ }^{1}}{{ }^{(1)} B_{k}{ }^{1}} \mathrm{~F}_{1 \mathrm{k}}\left[\begin{array}{ll}
i & j \\
i & j
\end{array}\right]=\left(\mathrm{F}_{\mathrm{k} 1}\left[\begin{array}{ll}
i & i \\
j & j
\end{array}\right]\right)^{-1}
$$

Substituting (17) back into (13) and taking $\ell=1$ 年 we recover the classifying algebra for boundary conditions (i.e. the Pasquier Algebra) [8 10, in the diagonal case:

$$
{ }^{(a)} B_{i}{ }^{1(a)} B_{j}{ }^{1}=\sum_{k} \mathrm{~N}_{i j}{ }^{k(a)} B_{k}{ }^{1}
$$

The general solution is given by the well known eigenvalues of the fusion matrices $\mathrm{N}_{i}$. Another way to obtain the same solution is to first find the general expression for the bulk-boundary couplings by considering (12) with $a=1, p=q=b$ (the $k$-sum reduces to $k=1)$ :

$$
{ }^{(b)} B_{i}^{\ell} C_{b \ell}^{(1 b b) b}={ }^{(1)} B_{i}{ }^{1} \sum_{m} e^{i \pi\left(2\left(h_{m}-h_{b}-h_{i}\right)+\frac{1}{2} h_{\ell}\right)} \mathrm{F}_{1 \mathrm{~m}}\left[\begin{array}{ll}
b & i \\
b & i
\end{array}\right] \mathrm{F}_{\mathrm{m} \ell}\left[\begin{array}{ll}
i & i \\
b & b
\end{array}\right]
$$

The constant ${ }^{(1)} B_{i}{ }^{1}$ is fixed by the normalisation of the bulk fields and the imaginary part of the sum can be shown to vanish, so that RHS is known and real. Petkova has observed [18] that up to a normalisation the ${ }^{(a)} B_{i}{ }^{k}$ are just the S-matrix elements $S_{a}{ }^{i}(k)$ for the torus with one operator insertion! This S-Matrix is given by equation (52) in the appendix. For $k=1$ this reduces to the S-matrix that implements the modular transformation of characters: $S_{i}{ }^{j}(1)=S_{i}{ }^{j}$.

Taking (19) and rearranging terms such that a rescaling invariant combination of structure constants appears on the LHS, together with (50)-(52) the correspondence between ${ }^{(a)} B_{i}{ }^{k}$ and $S_{a}{ }^{i}(k)$ becomes:

$$
\frac{{ }^{(a)} B_{i}{ }^{k} C_{a k}^{(1 a a) a}}{{ }^{(1)} B_{i}{ }^{1}}=e^{i \frac{\pi}{2} h_{k}}\left(\mathrm{~F}_{\mathrm{a} 1}\left[\begin{array}{ll}
a & a \\
k & k
\end{array}\right]\right)^{-1} \cdot \frac{S_{1}{ }^{1} \cdot S_{a}{ }^{i}(k)}{S_{1}{ }^{i} \cdot S_{a}{ }^{1}}
$$

This expression is symmetric under the exchange $a \leftrightarrow i$ and, since the phase on the RHS cancels with the phases in $S_{a}{ }^{i}(k)$, it is also real. Using the explicit normalisations ${ }^{(1)} B_{i}{ }^{1}=S_{i}{ }^{1} / S_{1}{ }^{1}$ and $C_{a k}^{(1 a a) a}=1$ (see equation (31) later on) (20) simplifies to

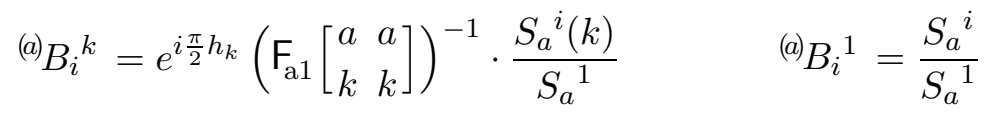

All the bulk and bulk-boundary structure constants are now determined and the scaling of all bulk fields is fixed.

\footnotetext{
${ }^{5}$ proper behaviour of the identity ensures that the appearing boundary structure constant is equal to one (see also (23) later on).

6 see also 19] for a general discussion of the relation between the sets of duality relations in [11] and 12
} 


\section{Boundary Structure Constants}

To give necessary expressions for the boundary structure constants it is enough only to consider equation (11). The following table summarises the line of argument in this section by describing the boundary situations considered, which constant it fixes, and what the remaining freedom to rescale the boundary fields is:

\begin{tabular}{|c|c|c|c|}
\hline boundary situation & s.c. fixed & eqn. & remaining freedom \\
\hline $\mathrm{b}_{\mathrm{b}}^{\mathrm{b}} \overbrace{\mathrm{j}}^{\mathrm{c}} \mathrm{b}_{\mathrm{b}}^{\mathrm{b}}$ & $\begin{array}{l}C_{b d}^{(b 1 d) q} \\
C_{\ell i}^{(d a b) q}\end{array}$ & $\begin{array}{l}\text { (29) } \\
\text { (31) }\end{array}$ & $\begin{array}{l}\begin{aligned} b \neq c, j \neq 1:\left\{\psi_{j}^{(b c)}, \psi_{j}^{(c b)}\right\} \rightarrow \\
$\[ \lambda=c, j \neq 1:\left\{\psi_{j}^{(b b)}\right\} \rightarrow\left\{ \pm \psi_{j}^{(b c)}, \lambda^{-1} \cdot \psi_{j}^{(b b)}\right\} \]$\end{aligned} \\
\text { none } \\
\text { none }\end{array}$ \\
\hline
\end{tabular}

In order to simplify the notation when rescaling subsets of boundary operators it is helpful to introduce an ordering on the boundary conditions $(1)<\left(a_{1}\right)<\left(a_{2}\right)<\ldots$ The particular order one chooses is not important.

As a first step consider the relation coming from taking the two different OPE's in the 3-point function:

$$
C_{i j}^{(a b c) k} C_{k k}^{(a c a) 1}=C_{j k}^{(b c a) i} C_{i i}^{(a b a) 1}
$$

Setting $i=1, k=j, b=a$ in (22) leads to $C_{1 j}^{(a a c) j}=C_{11}^{(a a a) 1}$. Similarly, setting $j=1$ resp. $k=1$ leads to $C_{i 1}^{(a b b) i}=C_{1 i}^{(b b a) i}$ resp. $C_{11}^{(a a a) 1}=C_{11}^{(b a a) i}$. It follows that consistent behaviour of the identity field on the 1-boundary $C_{11}^{(111) 1}=1$ already implies consistent behaviour of the identity on all other boundaries:

$$
C_{1 i}^{(a a b) i}=1 \quad C_{i 1}^{(a b b) i}=1 \quad \forall a, b, i
$$

Any given solution of the sewing constraints (11)-(15) must already fulfill (23).

To see how boundary operators couple to the identity, first consider (11) with $a=1, d=b, i=\ell=b, j=k, q=1$ : The sum reduces to $p=c$ and with (22) in the form $C_{j b}^{(c b 1) c} C_{c c}^{(1 c 1) 1}=C_{c j}^{(1 c b) b} C_{b b}^{(1 b 1) 1}$ one obtains:

$$
C_{j j}^{(b c b) 1}\left(\mathrm{~F}_{\mathrm{c} 1}\left[\begin{array}{ll}
j & j \\
b & b
\end{array}\right]\right)^{-1}=C_{b j}^{(1 b c) c} C_{c j}^{(1 c b) b}
$$

Exchanging $b \leftrightarrow c$ leaves the RHS invariant. Transforming the F-matrix element using (49) we finally get:

$$
S_{1}^{c} C_{j j}^{(c b c) 1}=S_{1}^{b} C_{j j}^{(b c b) 1}
$$

Any solution has to fulfill this identity. In particular this implies that independent of the expectation values of the identity $\langle 1\rangle^{(a)}$ we cannot set both $C_{j j}^{(c b c) 1}$ and $C_{j j}^{(b c b) 1}$ to one. 
We shall now make use of the freedom to rescale the fields. Note that the identity field 1 is fixed by the property $1 \cdot 1=1$. For $b<c$ and $j \neq 1$ we rescale $\psi_{j}^{(b c)} \rightarrow \lambda \cdot \psi_{j}^{(b c)}$. By (9) this results in:

$$
C_{j j}^{(c b c) 1} \rightarrow \lambda C_{j j}^{(b b c) 1} \quad C_{j j}^{(b c b) 1} \rightarrow \lambda C_{j j}^{(b c b) 1}
$$

For an appropriate $\lambda$ we get, for all $b<c, j \neq 1$ :

$$
C_{j j}^{(c b c) 1}=\left(F_{1 j}\left[\begin{array}{ll}
c & b \\
c & b
\end{array}\right]\right)^{-1} \frac{S_{1}^{1}}{S_{1}^{c}}
$$

In particular (26) implies that once the $C_{j j}^{(b b c) 1}$ are adjusted for $b<c$ one is no longer free to rescale $C_{j j}^{(\mathrm{coc}) 1}$ for $b>c$. However (25) implies that (27) holds also for $b>c, j \neq 1$. For $b=c, j \neq 1$ rescaling $\psi_{j}^{(b b)} \rightarrow \lambda \cdot \psi_{j}^{(b b)}$ gives $C_{j j}^{(b b b) 1} \rightarrow \lambda^{2} C_{j j}^{(b b b) 1}$, so that bringing $C_{j j}^{(b b b) 1}$ to the form (27) only fixes $\psi_{j}^{(b b)}$ up to a sign. Setting $j=1$, together with (47) shows consistency with $C_{11}^{(b b b) 1}=1$. Thus (27) is valid for all values of $b, c, j$. The scaling of the operators $\left\{\psi_{j}^{(b c)}, \psi_{j}^{(b)}\right\}$ for $b \neq c, j \neq 1$ is now fixed up to $\left\{\psi_{j}^{(b c)}, \psi_{j}^{(c b)}\right\} \rightarrow\left\{\lambda \cdot \psi_{j}^{(b c)}, \lambda^{-1} \cdot \psi_{j}^{((b)}\right\}$, which leaves (27) invariant. $\psi_{j}^{(b b)}, j \neq 1$ is fixed up to sign.

Taking (11) with $a=c=1, i=j=b, k=\ell=d, p=1$ and using (22) and (27) results in:

$$
C_{b d}^{(b 1 d) q} C_{d b}^{(d 1 b) q}=\left(\mathrm{F}_{1 \mathrm{q}}\left[\begin{array}{ll}
d & b \\
d & b
\end{array}\right]\right)^{2}
$$

For $b>d, q \neq 1$ we rescale $\left\{\psi_{q}^{(d b)}, \psi_{q}^{(b d)}\right\} \rightarrow\left\{\lambda \cdot \psi_{q}^{(d b)}, \lambda^{-1} \cdot \psi_{q}^{(b d)}\right\}$ such that:

$$
C_{b d}^{(b 1 d) q}=\mathrm{F}_{1 \mathrm{q}}\left[\begin{array}{ll}
d & b \\
d & b
\end{array}\right]
$$

(28) now implies that (29) also holds for $b<d$. For $b=d, q \neq 1$ we are still free to choose the sign of $\psi_{q}^{(b b)} \rightarrow \pm \psi_{q}^{(b b)}$. This allows us to alter the sign of $C_{b b}^{(b 1 b) q}$ to match (29). The case $q=1$ can occur only for $b=d$ and we get $C_{b b}^{(b 1 b) 1}=S_{1}{ }^{1} / S_{1}{ }^{b}$, consistent with the normalisation (27). Thus (29) holds for all $b, d, q$. The scaling of all boundary operators is now fixed.

Taking (11) with $c=1, j=b, k=d$ the sum reduces to $p=a$. Using (22) and rearranging terms one obtains:

$$
C_{\ell i}^{(d a b) q}=\mathrm{F}_{\mathrm{aq}}\left[\begin{array}{ll}
b & d \\
i & \ell
\end{array}\right] \frac{C_{i i}^{(a b a) 1} C_{\ell \ell}^{(d a d) 1}}{C_{q q}^{(d b d) 1} C_{a a}^{(a 1 a) 1}} \frac{C_{b a}^{(b 1 a) i} C_{a d}^{(a 1 d) \ell}}{C_{b d}^{(b 1 d) q}}
$$

All the C-terms cancel and after renaming indices one is left with:

$$
C_{i j}^{(a b c) k}=\mathrm{F}_{\mathrm{bk}}\left[\begin{array}{cc}
a & c \\
i & j
\end{array}\right] \quad \forall a, b, c, i, j, k
$$

Equations (17), (19) and (31) are the unique solution (if it exists) to the sewing constraints. All other solutions are trivially related by a rescaling of the fields. 


\section{One Point Function of the Identity Operator}

Recall that the 1-pt-function of the 1-operator cancelled in equation (11)-(13) (and in (14), (15) as well in fact). We can fix these by demanding that the partition function around a cylinder of length $R$ and circumfence $L$ is a modular transformation of the partition function along the cylinder [10,20].

The partition function around the cylinder is equivalent to a trace on the UHP which reduces to a sum of characters:

$$
Z_{(a b)}[\underline{\mathrm{a}} \underline{\mathrm{b}}]=\sum_{j} n_{j a}{ }^{b} \chi_{j}(q)=\sum_{i, j} \frac{S_{a}{ }^{i} S_{b}{ }^{i}}{S_{1}{ }^{i}} \cdot S_{i}{ }^{j} \chi_{j}(q)=\sum_{i} \frac{S_{a}{ }^{i} S_{b}{ }^{i}}{S_{1}{ }^{i}} \cdot \chi_{i}(\tilde{q})
$$

where $q=\exp (-\pi L / R)$ and $\tilde{q}=\exp (-4 \pi R / L)$.

The partition function along the cylinder in turn is equivalent to an inner product of boundary states. Let $|i\rangle\rangle=\left(1+\left(2 h_{i}\right)^{-1} L_{-1} \bar{L}_{-1}+\cdots\right)|i\rangle$ denote the Ishibashi states [21] and $\left.|a\rangle=\sum_{i} g_{a i}|i\rangle\right\rangle$ be a boundary state. It will turn out that there is a consistent choice of constants such that the $g_{a i}$ are real and complex conjugation has thus been left out of subsequent formulas. For the partition function we get:

$$
Z_{(a b)}[\text { (a) } \mathrm{b}]=\left\langle b\left|e^{-\frac{2 \pi R}{L}\left(L_{0}+\bar{L}_{0}-\frac{c}{12}\right)}\right| a\right\rangle=\sum_{i} g_{b i} g_{a i}\langle i \mid i\rangle \cdot \chi_{i}(\tilde{q})
$$

Comparing (33) to (32) gives expressions for $g_{a i}$. Alternatively these can be obtained by calculating the 1-pt-function on a disc in two different ways. Consider a bulk field $\phi_{i}$

in the centre of a disc of radius 1 with boundary condition $b$. Using Ishibashi states one gets:

$$
\left\langle\phi_{i}(0)\right\rangle_{\text {disc }}^{(b)}=\left\langle b\left|\phi_{i}(0)\right| 0\right\rangle=g_{b i}\langle i \mid i\rangle
$$

Here we took the states $|i\rangle$ to be normalised such that:

$$
\phi_{i}(0)|0\rangle=|i\rangle
$$

On the other hand, transforming the the 1-pt-function from the UHP with a $b$-boundary to the disc we get:

$$
\left\langle\phi_{i}(0)\right\rangle_{\mathrm{disc}}^{(b)}={ }^{(b)} B_{i}{ }^{1}\langle 1\rangle^{(b)}
$$

Comparing the two yields:

$$
g_{b i}=\frac{{ }^{(b)} B_{i}{ }^{1}\langle 1\rangle^{(b)}}{\langle i \mid i\rangle}
$$

The norm of the state $|i\rangle$ is linked to OPE of two bulk fields via (35):

$$
\langle i \mid i\rangle=C_{i i}{ }^{1}\langle 0 \mid 0\rangle
$$

To ensure that the boundary 1-pt-functions of the identity are real and take a concise form we choose not to set $\langle 0 \mid 0\rangle$ to one. 
Substituting (37) back into (33) gives:

$$
g_{a i} g_{b i}\langle i \mid i\rangle=\frac{{ }^{(a)} B_{i}{ }^{(b)} B_{i}{ }^{1}}{C_{i i}{ }^{1}} \cdot \frac{\langle 1\rangle^{(a)}\langle 1\rangle^{(b)}}{\langle 0 \mid 0\rangle}
$$

The RHS of this equation is invariant under a rescaling $\phi_{i} \rightarrow \lambda \phi_{i}$ because the factors from ${ }^{(a)} B_{i}{ }^{1} \cdot{ }^{(b)} B_{i}{ }^{1}$ and $C_{i i}{ }^{1}$ cancel. Thus substituting the explicit expressions for ${ }^{(b)} B_{i}{ }^{1}$ (21) and $C_{i i}{ }^{1}$ (17) still gives an expression that is invariant under rescaling of the fields. Comparing (33) to (32) now yields:

$$
\frac{\langle 1\rangle^{(a)}\langle 1\rangle^{(b)}}{\langle 0 \mid 0\rangle}=\frac{S_{a}{ }^{1} S_{b}{ }^{1}}{S_{1}{ }^{1}}
$$

The general solution to (40) is:

$$
\langle 1\rangle^{(a)}=\mu \cdot S_{a}{ }^{1} \quad\langle 0 \mid 0\rangle=\mu^{2} \cdot S_{1}{ }^{1}
$$

For convenience we choose $\mu=1$.

There is another constraint on the identity 1-pt-functions, coming from demanding continuity on the disc. We take boundary fields to transform as $\psi_{i}^{(a b)}(x) \rightarrow\left|\varphi^{\prime}(x)\right|^{h_{i}} \psi_{i}^{(a b)}(\varphi(x))$. If we lift the two UHP 2-pt-functions $\left\langle\psi_{i}^{(a b)}(x) \psi_{i}^{(b a)}(y)\right\rangle_{U H P}^{(a)}$ and $\left\langle\psi_{i}^{(b a)}(y) \psi_{i}^{(a b)}(x)\right\rangle_{U H P}^{(b)}$ to the disc, they should be identical upon analytic continuation. This results in the constraint (see also [11]):

$$
C_{i i}^{(a b a) 1}\langle 1\rangle^{(a)}=C_{i i}^{(b a b) 1}\langle 1\rangle^{(b)}
$$

Comparing (42) with (25), independent of the normalisation of boundary fields, this becomes:

$$
\frac{\langle 1\rangle^{(a)}}{S_{1}^{a}}=\frac{\langle 1\rangle^{(b)}}{S_{1}^{b}}
$$

The general solution for $\langle 1\rangle^{(a)}$ in (41) is thus consistent with demanding continuity on the disc, for any value of $\mu$.

One can now work out the universal ground state degeneracies defined in [22]:

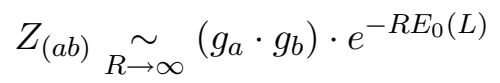

where $E_{0}(L)$ is the ground state energy and $g_{a}$ resp. $g_{b}$ is the factor of the ground state degeneracy coming from the boundary $a$ resp. $b$. Let $\phi_{\Omega}$ be the field of lowest conformal weight. Then the S-matrix (for Virasoro minimal models) satisfies $S_{\Omega}{ }^{a}>0 \forall a$. From (33) we get the relation $g_{a} \cdot g_{b}=g_{a \Omega} \cdot g_{b \Omega} \cdot\langle\Omega \mid \Omega\rangle$. Thus

$$
g_{a}=g_{a \Omega} \sqrt{\langle\Omega \mid \Omega\rangle}=\frac{{ }^{(a)} B_{\Omega}{ }^{1} \cdot\langle 1\rangle^{(a)}}{\sqrt{C_{\Omega \Omega}{ }^{1}\langle 0 \mid 0\rangle}}=\frac{S_{a}{ }^{\Omega}}{\sqrt{S_{1}^{\Omega}}}
$$

in agreement with [22]. Note that this expression is invariant under both rescaling of the fields and rescaling of the identity-1-pt-functions. 


\section{Conclusion}

In this paper we have found the structure constants of A-type Virasoro minimal models, given in equations (17), (19) and (31), under the assumption that a solution to the sewing constraints exists. Any other solution is trivially related to the given set of structure constants by a rescaling of the fields. Numerical tests with randomly chosen fields in several A-type Virasoro minimal models confirm that the given structure constants solve the sewing constraints (11)-(15).

In the form given in section 旬 the sewing constraints do not depend on the boundary 1pt-function of the identity $\langle 1\rangle^{(a)}$. In particular there is no normalisation of the boundary fields s.t. for $a \neq b$ we can have both $C_{i i}^{(a b a) 1}=1$ and $C_{i i}^{(b a b) 1}=1$ (see equation (25)). Also none of the structure constants given depends on the final choice for the values of $\langle 1\rangle^{(a)}$.

There are two constraints on the $\langle 1\rangle^{(a)}$ and the normalisation of the bulk-vacuum $\langle 0 \mid 0\rangle$ : one from modular transformation of the partition function (40) and one from demanding continuity on the disc (43). In fact these are equivalent, because on the one hand, once $\langle 0 \mid 0\rangle$ is chosen, (40) actually determines the $\langle 1\rangle^{(a)}$ up to an overall sign and one can check that (43) is satisfied, irrespective of the choice of that sign. On the other hand, once (43) is solved, we can choose $\langle 0 \mid 0\rangle$ such that (40) is also satisfied. The choice for $\langle 1\rangle^{(a)}$ and $\langle 0 \mid 0\rangle$ is not affected by rescaling of the bulk or boundary fields.

The individual rescalings are, for $\alpha_{i}, \beta_{i}^{(a b)}, \mu \in \mathbb{C}$ :

$$
\begin{aligned}
\phi_{i} & \rightarrow \alpha_{i} \cdot \phi_{i} \\
\psi_{i}^{(a b)} & \rightarrow \beta_{i}^{(a b)} \cdot \psi_{i}^{(a b)} \\
\langle 0 \mid 0\rangle,\langle 1\rangle^{(a)},\langle 1\rangle^{(b)}, \ldots & \rightarrow \mu^{2}\langle 0 \mid 0\rangle, \mu\langle 1\rangle^{(a)}, \mu\langle 1\rangle^{(b)}, \ldots
\end{aligned}
$$

To relate the normalisation used in this paper to others one can look at the scale dependant form of the following structure constants:

$$
{ }^{(a)} B_{i}{ }^{1}=\alpha_{i} \cdot \frac{S_{a}{ }^{i}}{S_{a}{ }^{1}} \quad C_{i i}{ }^{1}=\left(\alpha_{i}\right)^{2} \cdot \frac{S_{1}{ }^{i}}{S_{1}{ }^{1}} \quad\langle 0 \mid 0\rangle=\mu^{2} \cdot S_{1}{ }^{1} \quad\langle 1\rangle^{(a)}=\mu \cdot S_{a}{ }^{1}
$$

In this paper we have $\alpha_{i}=1, \mu=1$. To recover the normalisation of Cardy and Lewellen [7, 11] one has to set $\alpha_{i}=\sqrt{S_{1}{ }^{1} / S_{1}{ }^{i}}$ and $\mu=1 / \sqrt{S_{1}{ }^{1}}$.

The ground state degeneracies have been computed in this formalism and the result agrees with [22] and is, as expected, invariant under all rescalings.

Acknowledgements - This work was supported by the EPSRC, the DAAD and King's College London. I especially wish to thank my supervisor G.M.T. Watts for suggesting the project, encouragement, criticism and helpful discussions, as well as for sharing the fruits of conversations with J.-B. Zuber at meetings in SISSA, DESY and Durham. Furthermore I want to thank V.B. Petkova for several useful conversations and I am grateful to her, A. Sagnotti, Ya.S. Stanev and J.-B. Zuber for comments and corrections to early versions of this paper. 


\section{Appendix: Some Useful Identities for F-Matrices}

The following identities from [12 for Virasoro minimal models are useful when working

with the structure constants:

$$
\begin{aligned}
& \mathrm{F}_{\mathrm{pq}}\left[\begin{array}{cc}
j & k \\
i & \ell
\end{array}\right]=\mathrm{F}_{\mathrm{pq}}\left[\begin{array}{ll}
i & \ell \\
j & k
\end{array}\right]=\mathrm{F}_{\mathrm{pq}}\left[\begin{array}{ll}
\ell & i \\
k & j
\end{array}\right] \\
& \mathrm{F}_{11}\left[\begin{array}{ll}
i & i \\
i & i
\end{array}\right]=\frac{S_{1}{ }^{1}}{S_{1}{ }^{i}} \\
& \mathrm{~F}_{1 \mathrm{k}}\left[\begin{array}{cc}
i & j \\
i & j
\end{array}\right] \mathrm{F}_{\mathrm{k} 1}\left[\begin{array}{ll}
i & i \\
j & j
\end{array}\right]=\frac{S_{1}{ }^{1} \cdot S_{1}{ }^{k}}{S_{1}{ }^{i} \cdot S_{1}{ }^{j}} \\
& \mathrm{~F}_{\mathrm{k} 1}\left[\begin{array}{cc}
i & i \\
j & j
\end{array}\right]=\frac{S_{1}^{k}}{S_{1}^{j}} \cdot \mathrm{F}_{\mathrm{j} 1}\left[\begin{array}{cc}
k & k \\
i & i
\end{array}\right] \\
& \mathrm{F}_{\mathrm{n} 1}\left[\begin{array}{ll}
i & i \\
\ell & \ell
\end{array}\right] \mathrm{F}_{\mathrm{p} i}\left[\begin{array}{ll}
j & k \\
n & \ell
\end{array}\right]=\mathrm{F}_{\mathrm{p} 1}\left[\begin{array}{ll}
k & k \\
\ell & \ell
\end{array}\right] \mathrm{F}_{\mathrm{nk}}\left[\begin{array}{ll}
i & j \\
\ell & p
\end{array}\right] \\
& \mathrm{B}_{p q}^{(\epsilon)}\left[\begin{array}{ll}
j & k \\
i & \ell
\end{array}\right]=e^{i \pi \epsilon\left(h_{i}+h_{\ell}-h_{p}-h_{q}\right)} \mathrm{F}_{\mathrm{pq}}\left[\begin{array}{ll}
j & l \\
i & k
\end{array}\right]
\end{aligned}
$$

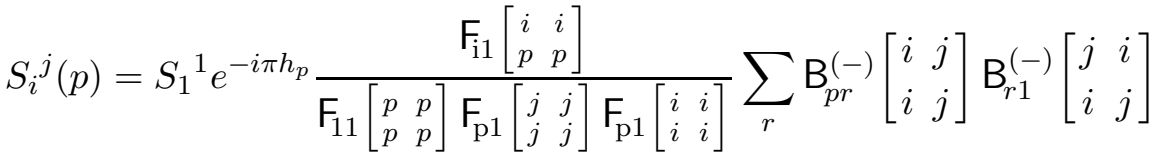

\section{References}

[1] A.A. Belavin, A.M. Polyakov, A.B. Zamolodchikov, Infinite conformal symmetry in two-dimensional quantum field theory, Nucl. Phys. B241 (1984) 333-380.

[2] Ph. Di Francesco, P. Mathieu, D. Sénéchal, Conformal Field Theory, Springer 1998.

[3] A. Capelli, C. Itzykson, J.-B. Zuber, Modular invariant partition functions in two dimensions, Nucl. Phys. B280 (1987) 445-465.

[4] V.P. Yurov, A.B. Zamolodchikov, Truncated conformal space approach to scaling Lee-Yang model, Int. J. Mod. Phys. A5 (1990) 3221-3246.

[5] P. Dorey, A. Pocklington, R. Tateo, G. Watts, TBA and TCSA with boundaries and excited states, Nucl. Phys. B525 (1998) 641-663, hep-th/9712197. P. Dorey, I. Runkel, R. Tateo, G. Watts, in preparation

[6] J.L. Cardy, Conformal invariance and surface critical behavior, Nucl. Phys. B240 (1984) $514-532$.

[7] J.L. Cardy, D.C. Lewellen, Bulk an boundary operators in conformal field theory, Phys. Lett. B259 (1991) 274-278.

[8] G. Pradisi, A. Sagnotti, Ya.S. Stanev, Completeness conditions for boundary operators in 2-D conformal field theory, Phys. Lett. B381 (1996) 97-104, hep-th/9603097.

[9] J. Fuchs, C. Schweigert, A Classifying algebra for boundary conditions, Phys. Lett. B414 (1997) 251-259, hep-th/9708141.

[10] R.E. Behrend, P.A. Pearce, J.-B. Zuber, Integrable boundaries, conformal boundary conditions and A-D-E fusion rules, J. Phys. A31 (1998) L763-L770, hep-th/9807142. R.E. Behrend, P.A. Pearce, V.B. Petkova, J.-B. Zuber, On the Classification of Bulk and Boundary Conformal Field Theories, Phys. Lett. B444 (1998) 163-166, hep-th/9809097. 
[11] D.C. Lewellen, Sewing constraints for conformal field theories on surfaces with boundaries, Nucl. Phys. B372 (1992) 654-682.

[12] G. Moore and N. Seiberg, Lectures on RCFT, Physics, Geometry, and Topology, Plenum Press, New York, 1990.

[13] Vl.S. Dotsenko, V.A. Fateev, Conformal algebra and multipoint correlation functions in $2 d$ statistical models, Nucl. Phys. B240 (1984) 312-348. Four-point correlation functions and the operator agebra in 2d conformal invariant theories with central charge $c \leq 1$, Nucl. Phys. B251 (1985) 691-734.

[14] D. Fioravanti, G. Pradisi, A. Sagnotti, Sewing constraints and nonorientable open strings, Phys. Lett. B321 (1994) 349-354, hep-th/9311183. G. Pradisi, A. Sagnotti, Ya. S. Stanev, Planar duality in SU(2) WZW models Phys. Lett. B354 (1995) 279-286, hep-th/9503207. The open descendants of nondiagonal SU(2) WZW models Phys. Lett. B356 (1995) 230-238, hep-th/9506014.

[15] L. Alvarez-Gaumé, C. Gomez, G. Sierra, Quantum group interpretation of some conformal field theories, Phys. Lett. B220 (1989) 142-152.

[16] P. Furlan, A.Ch. Ganchev, V.B. Petkova, Fusion matrices and $c<1$ (quasi) local conformal field theories, Int. J. Mod. Phys. A5 (1990) 2721-2735.

[17] G. Felder, J. Fröhlich, G. Keller, Braid matrices and structure constants for minimal conformal models, Comm. Math. Phys. 124 (1989) 647-664.

[18] V.B. Petkova, private communication

[19] R.E. Behrend, P.A. Pearce, V.B. Petkova, J.-B. Zuber, to appear

[20] J.L. Cardy, Boundary conditions, fusion rules and the Verlinde formula, Nucl. Phys. B324 (1989) 581-596.

[21] N. Ishibashi, The boundary and crosscap states in conformal field theories, Mod. Phys. Lett. A4: 251 (1989)

[22] I. Affleck, A.W.W. Ludwig, Universal Noninteger "Ground-State Degeneracy" in Critical Quantum Systems, Phys. Rev. Lett. 67 (1991) 161-164. 